\title{
Guest editorials: P2P computing for 5G, beyond 5G (B5G) networks and internet-of-everything (IoE)
}

\author{
P. Prakasam ${ }^{1} \cdot$ Md. Shohel Sayeed ${ }^{2} \cdot$ J. Ajayan ${ }^{3}$ \\ Published online: 16 September 2020 \\ (C) Springer Science+Business Media, LLC, part of Springer Nature 2020
}

Due to the technology advancements, in recent days, most of the wireless devices are connected to machines (IoT) rather than human being, will lead to the growth in Smart Cities, Smart Homes, Smart Vehicles etc. Hence, the future customers will request for higher capacity, higher data rate with global connectivity. Hence, 5G and B5G networks encounter fundamental challenges for peer-to-peer IoT communications, including automated vehicle, robotic and other automatic systems, and computing infrastructures. Therefore Internet-ofEverything (IoE), integration of everything in a single hub is the related development. Other challenges predicted for future networks are to handle Ultra-dense cell networks, Reconfigurable Hardware, Networked VLC, Networking Intelligence and technological developments to empower the users with $100 \%$ Immersive Experiences.

It is anticipated that P2P Computing and Blockchain with AI will be the very significant technologies for an effective development of the future wireless networks. Since, P2P and Blockchain technologies incorporates a distributed network concepts to provide secure services without any Cluster head, due to this distributed nature, P2P and Blockchain will be a suitable

This article is part of the Topical Collection: Special Issue on P2P Computing for Beyond $5 G$ Network and Internet-of-Everything Guest Editors: Prakasam P, Ajayan John, Shohel Sayeed

P. Prakasam

prakasamp@gmail.com

Md. Shohel Sayeed

shohel.sayeed@mmu.edu.my

J. Ajayan

email2ajayan@gmail.com

1 School of Electronics Engineering, Vellore Institute of Technology, Vellore, Tamil Nadu 632014, India

2 Faculty of Information Science and Technology, Multimedia University, 75450 Melaka, Malaysia

3 Department of Electronics and Communication Engineering, SNS College of Technology, Coimbatore, Tamil Nadu 641035, India technology for dynamic spectrum sharing in $5 \mathrm{G}$ and B5G. Also along with these two technologies, AI approaches like machine learning and deep learning can be combined and deployed into $5 \mathrm{G}$ and $\mathrm{B} 5 \mathrm{G}$ networks to collect, analyse the data and manage the available resources in an efficient manner.

The aim of this special issue is to focus the challenges, case studies, architectures, algorithms and application for $\mathrm{P} 2 \mathrm{P}$ computing and blockchain enabled 5G, B5G networks and IoE.

The special issue has declared as an open call for papers and rigorous peer-review has been carried. Totally 31 research articles have been received for this SI and 13 of them have been accepted after peer review as a witness of ongoing research ideas and solutions related to B5G and IoE.

The first article by Steffi Jayakumar, Nandakumar, S. on 'A review on resource allocation techniques in D2D communication for $5 \mathrm{G}$ and $\mathrm{B} 5 \mathrm{G}$ technology' studied the various challenges faced by $5 \mathrm{G}$ and $\mathrm{B} 5 \mathrm{G}$ wireless networks. Based on the survey, the resource allocation algorithms and methodologies have been seriously analysed and evaluated to find the research gap and solution for the research problems related to resource allocation in D2D communication.

The second article by Senthil Kumar, A. Thirunavukkarasu, $V$. on 'Peer to peer communication framework for $5 \mathrm{G}$ networks using Ambient Service' proposed a Peer to Peer Communication Framework along with Ambient Service implementation for $5 \mathrm{G}$ networks to support Radio Access Network (RAN).

The third article by Divya Mohan, Geetha Mary, A. on 'The Efficacy of Handoff Strategy Based Spectrum Allocation Using TSB-MFO For D2D and CU Communications' developed an efficient handoff strategy based on bandwidth allocation using Thompson Sampling Bandwidth (TSB) and Magnetic Force Optimization (MFO) for D2D and CU communications without assuming Channel State Information (CSI) at the base station (BS).

The fourth article by Vali Mohamad et al. on 'Dynamic Sectorization and parallel processing for device-to-device (D2D) resource allocation in 5G and B5G cellular network' presented a dynamic sectorization technique in which eNodeB (eNB) varies the number of sectors dynamically in the 
network and allocates the Resource Block (RB) to D2D users. Also they have derived an expression for the probability of successful transmission and threshold to make a decision on the number of sectors based on available RBs and D2D users in the network.

The fifth article by Bilim, $M$. on 'Dual-branch SC wireless systems with HQAM for beyond $5 \mathrm{G}$ over $\eta-\mu$ fading channels' analysed the performance of hexagonal quadrature amplitude modulation (HQAM) for a dual-branch selection combining (SC) receiver for beyond fifth-generation (B5G) with $\eta-\mu$ fading channels in a detailed manner.

The sixth article by Christopher Clement et al. on 'Deep learning based modulation classification for $5 \mathrm{G}$ and beyond wireless systems' proposed a modulation classification algorithm using the combined architectures of modified convolutional neural network. Further, they have developed a deep learning architecture by combining the convolutional neural network, dense network, and long short-term memory network (LSTM), which is named as convolutional LSTM dense neural network (CLDNN).

The seventh article by Malini, B. Noor Mohammed, V. on 'Joint Power Optimization and Scaled Beamforming Approach in B5G Network Based Massive MIMO Enabled HetNet with Full-duplex Small Cells' investigated the power optimization technique for B5G network using the macro base station enabled with massive MIMO technology and the small cell access points possessing the fullduplex communication ability in Heterogeneous Networks (HetNets).

The eighth article by Acharjya, D.P. Syed Siraj Ahmed, N. on 'Tracing of online assaults in 5G networks using dominance based rough set and formal concept analysis' developed a model to recognize various assaults via online in $5 \mathrm{G}$, B5G and IoE networks using dominance based rough set and formal concept analysis. Further they have analysed using various types of assaults and the assaults have been identified using the dominance based rough set.

The ninth article by Reshmi, T.R. Azath, M. on 'Improved self-healing technique for $5 \mathrm{G}$ networks using predictive analysis' introduced an automated network diagnostics and self-healing technique for $5 \mathrm{G}$ environment using predictive analysis. The data has been collected by considering the performance parameters of the device or network and if the performance parameters are deviated from the normal ranges then the problems occurred in the network are diagnosed in a productive way.

The tenth article by Sankar, S.P. et al. on 'Security improvement in block chain technique enabled peer to peer network for beyond $5 \mathrm{G}$ and internet of things' implemented a data security by employing private blockchain in SDN and public blockchain for peer to peer communication. Also they have proposed a secured authentication method to validate the blocks in the network.
The eleventh article by Vivekanandan, M. Sastry, V. N. Reddy S.U. on 'BIDAPSCA5G: Blockchain based Internet of Things (IoT) device to device authentication protocol for smart city applications using 5G technology' proposed a Blockchain based Internet of Things (IoT) Device to Device Authentication Protocol for Smart City Applications using 5G Technology (BIDAPSCA5G) in which the IoT Devices registration process is performed through private blockchain. The mutual authentication has been accomplished without involvement of RAC/Gate-Way-Node (GWN) to reduce the computation cost.

The twelfth article by Kumar, P. Chouhan, L. on 'A secure authentication scheme for IoT application in smart home' designed a smart card based secure addressing and authentication (SCSAA) scheme by modifying the standard IPv6 protocol to mitigate the security threats in the IoT network. The proposed scheme has two folds as it assigns an unique 64-bit interface identifier (IID) to smart devices/appliances and uniquely authenticates them in IoT network and also it uses the secret session key to prevent the network from unauthorized access.

The thirteenth article by Riya, Gupta, N. Dhurandher, S.K. on 'Efficient caching method in fog computing for internet of everything' suggested the methodology to reduce the latency in fog computing which will more suitable for IoE. Further this has been achieved by performing the popularity based caching. In this circumstances, initially the clustering of the IoT devices has been done on the basis of their interests and distance between them using spectral clustering technique and then each cluster is mapped with the fog and further to reduce the latency, in case of cache miss, the Device to Device (D2D) communication is used.

Based on the above research articles it has been found that many researchers are focusing on the security issues and resource allocation algorithm to meet the future demand for 5G, B5G wireless networks and Internet-ofEverything (IoE).

We would like to thank all the reviewers who have helped and reviewed the articles in time, and the authors for their potential contribution and efforts to complete this special issue with high quality even though in Corona-19 pandemic situation. Finally we would like to extent our gratitude to Prof. Xuemin (Sherman) Shen, Editor-in-Chief for accepting this special issue proposal and encouraging us to undertake this remarkable assignment. Also we would like to extent our sincere thanks to Ms. Melissa Fearon, Senior Publishing Editor, Springer, Katherine Moretti, Assistant Editor, Computer Science, Springer and Mr. Genesis Obero, Journal Editorial Office (JEO), Springer for their help during the publication process.

Publisher's note Springer Nature remains neutral with regard to jurisdictional claims in published maps and institutional affiliations. 


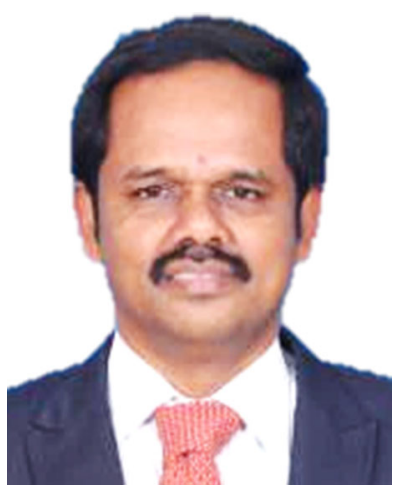

P. Prakasam is currently working as a Professor in School of Electronics Engineering, Vellore Institute of Technology, Vellore, India. He obtained his Ph.D in Signal Processing in Wireless Communication (Software Defined Radio) from Anna University Chennai in the year 2010. He is an Associate Editor of IEEE Access, Array (Elsevier) and also an editor-in-chief of Journal of Signal Processing and Wireless Networks. He has authored over 120 research publications in various journals and conferences out of which 35 publications have been indexed by SCI/SCIE/EI. He has more than 5 monographs/ books, 3 book chapters, 10 Indian patents, and 2 Best Paper Awards. He is a reviewer of more than 20 journals in various publishers which includes IEEE, Elsevier and Springer journals. He has received various awards which includes Best Faculty in Engineering. His name has been selected to include for Albert Nelson Marquis Lifetime Achievement Award, 2018 for outstanding achievements. He has served more than 20 conferences as technical advisory/reviewer committee. His special areas of interest are Signal Processing, Wireless Networks, Machine/Deep Learning, 5G Networks and applications of signal processing in Mobile Communication Systems. He is also Senior Member in IEEE and IACSIT, and life member of ISTE.

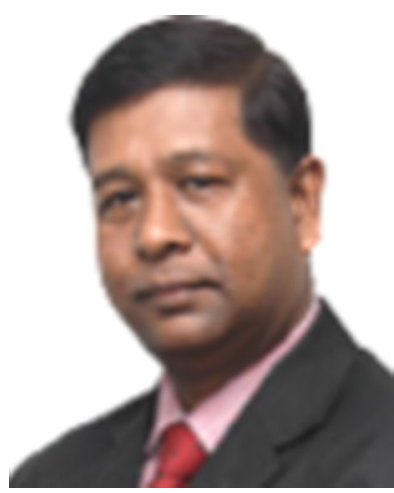

Md. Shohel Sayeed is currently working as an Associate Professor of the Faculty of Information Science and Technology, Multimedia University, Malaysia. His core research interest is in the area of Biometrics, information security, Wireless Sensor Networks, Signal processing, Wireless Communications, pattern recognition and classification. He has been awarded Marquis Who's who in the world in 2010 for his outstanding contribution. Also he has received the International President's Award for Iconic Achievement,
2010, by International Biographical Centre, Cambridge, England. Till date, he has published over 80 research papers in international peerreviewed journals and international conference proceedings. He has more than 2 books, 1 book chapter and 4 copyright/patent. He has undertaken more than 7 scientific research projects as the leader, including 5 nationallevel projects such as Fundamental Research Grant Scheme (FRGS). He is a reviewer of more than 10 journals in various publishers which includes IEEE, Elsevier and Springer journals. He has also been invited to review technical papers for several international conferences. He is an editorial board member for an edited book on Security and Authentication: Perspectives, Management and Challenges (2018). He is also a Member in IEEE and IACSIT.

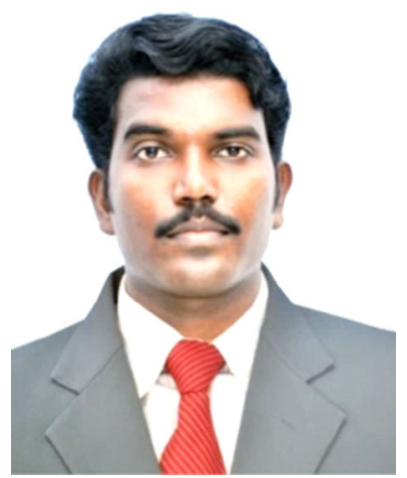

J. Ajayan is a senior assistant professor in the department of Electronics and Communication Engineering at SNS College of Technology, Coimbatore, India. He has published more than 70 research articles in various journals (Elsevier, Taylor and Francis, Springer, IOP Science) and international conferences. He has more than 2 monographs/ books, 1 book chapters, and 1 Indian patents. He is a reviewer of more than 30 journals in various publishers which includes IEEE, IET, Elsevier and Springer journals. He is also Guest Editor of "Special Issue on Energy Harvesting Devices, Circuits and Systems for Internet of Things" in Microelectronics Journal, Elsevier. He has served more than 10 conferences as technical advisory/reviewer committee. His special areas of interest are micro/nano scale devices for wireless applications, Machine/Deep Learning, Embedded systems and VLSI Design. 